\title{
Current perspectives on invasive nontyphoidal Salmonella disease
}

\author{
Andrea H. Haselbeck ${ }^{\mathrm{a}, *}$, Ursula Panzner ${ }^{\mathrm{a}, *}$, Justin $I m^{\mathrm{a}}$, Stephen Baker ${ }^{\mathrm{b}, \mathrm{c}}$, \\ Christian G. Meyer ${ }^{\mathrm{d}, \mathrm{e}}$, and Florian Marks ${ }^{\mathrm{a}, \mathrm{c}}$
}

\begin{abstract}
Purpose of review
We searched PubMed for scientific literature published in the past 2 years for relevant information regarding the burden of invasive nontyphoidal Salmonella disease and host factors associated with nontyphoidal Salmonella infection and discuss current knowledge on vaccine development. The following search terms were used: Salmonella, non typhoidal/nontyphoidal, NTS, disease, bloodstream infection, invasive, sepsis/septicaemia/septicemia, bacteraemia/bacteremia, gastroenteritis, incidence, prevalence, morbidity, mortality, case fatality, host/risk factor, vaccination, and prevention/control.
\end{abstract}

\section{Recent findings}

Estimates of the global invasive nontyphoidal Salmonella disease burden have been recently updated; additional data from Africa, Asia, and Latin America are now available. New data bridge various knowledge gaps, particularly with respect to host risk factors and the geographical distribution of iNTS serovars. It has also been observed that Salmonella Typhimurium sequence type 313 is emergent in several African countries. Available data suggest that genetic variation in the sequence type 313 strain has led to increased pathogenicity and human host adaptation. A bivalent efficacious vaccine, targeting Salmonella serovars Typhimurium and Enteritidis, would significantly lower the disease burden in high-risk populations.

\section{Summary}

The mobilization of surveillance networks, especially in Asia and Latin America, may provide missing data regarding the invasive nontyphoidal Salmonella disease burden and their corresponding antimicrobial susceptibility profiles. Efforts and resources should be directed toward invasive nontyphoidal Salmonella disease vaccine development.

\section{Keywords}

epidemiology, invasive nontyphoidal Salmonella disease, invasive, nontyphoidal Salmonella, vaccination

\section{INTRODUCTION}

Salmonella enterica serovar Typhi (S. Typhi) and the various pathovars of $S$. Paratyphi are commonly referred to as typhoidal Salmonella serovars. These agents are restricted to human hosts. Salmonella serovars that fall outside of this group are typically referred to as the nontyphoidal Salmonella (NTS) serovars and are considered to have the potential to interact with human and nonhuman hosts [1"']. Poor access to improved water supplies and adequate sanitation facilities, combined with growing urbanization, favor the transmission of NTS serovars through food or water sources and contact with animals [2"']. In addition to animal reservoirs, humans may be a growing substantial secondary pathogen reservoir [3]. Typical NTS disease in immunocompetent hosts manifests as a mild, selflimiting gastroenteritis. In contrast, invasive nontyphoidal Salmonella (iNTS) disease commonly presents as a febrile bacteremia, which can be fatal if left untreated. Invasive NTS disease is associated with the extremes of age, malnutrition, clinical malaria, and HIV infections, especially in Africa [4",5"--7"']. In this study, we review available literature published

anternational Vaccine Institute, Epidemiology Unit, Seoul, Republic of

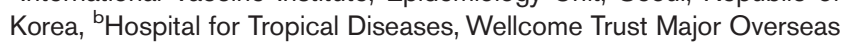
Programme, Oxford University Clinical Research Unit, Ho Chi Minh City, Vietnam, ' Department of Medicine, University of Cambridge, Cambridge, United Kingdom, ${ }^{d}$ Institute of Tropical Medicine, Eberhard-Karls University Tübingen, Tübingen, Germany and 'Duy Tan University, Da Nang, Vietnam Correspondence to Florian Marks, MPH, PhD, International Vaccine Institute, 1 Gwanak-ro, 1 Gwanak-gu, Seoul 151-742, Republic of Korea. Tel: +82 2881 1133; e-mail: fmarks@ivi.int

${ }^{*}$ Andrea H. Haselbeck and Ursula Panzner contributed equally to the writing of this article.

Curr Opin Infect Dis 2017, 30:498-503

DOI:10.1097/QC0.0000000000000398

This is an open access article distributed under the Creative Commons Attribution License 4.0 (CCBY), which permits unrestricted use, distribution, and reproduction in any medium, provided the original work is properly cited. 


\section{KEY POINTS}

- Global estimates suggest 3.4 million iNTS illnesses and 618316 iNTS disease-related deaths per year.

- The most common iNTS serovars are S. Enteritidis, S. Dublin, S. Typhimurium; of particular concern is the S. Typhimurium ST313 variant.

- Bivalent S. Enteritidis and S. Typhimurium vaccines may decrease the global disease burden dramatically.

from 2015 to present on the global burden of iNTS disease, host risk factors, and the implications of these data for vaccination.

\section{GLOBAL DISEASE BURDEN}

A recent review on the global distribution of iNTS has indicated a low number of reported cases in South and Southeast Asia [8"]. The overwhelming majority of the estimated 3.4 million annual iNTS infections and 618316 iNTS-related deaths occur in Africa [4",8*,9]. Salmonella enterica serovars Enteritidis, Dublin, and Typhimurium are the most common serovars associated with iNTS disease $\left[4^{*}, 5^{\boldsymbol{\prime}}\right]$. Of specific concern is $S$. Typhimurium sequence type 313 (ST313), which is more frequently associated with bacteremia than gastrointestinal infections $[10,11]$, and is commonly multidrug resistant (MDR) [2"',12]. Additional data suggest that $S$. Typhimurium ST313 may have a greater propensity for transmission between humans, and an animal reservoir has not, as yet, been well defined. Keddy et al. [13] recently found a significant association of an MDR phenotype in ST313 [odds ratio (OR) 6.6; $95 \%$ confidence interval $(95 \% \mathrm{CI}) 2.5-17.2]$ in comparison to $S$. Typhimurium sequence type 19 (ST19). ST19 arises mostly in Europe and Northern America, whereas ST313 isolates are more commonly found in Africa [14"'].

A review published in 2017 assessed the occurrence of iNTS disease across Africa [5"']. The authors described disease incidence estimates that ranged from 1.4 per 100000 population/year (all ages, South Africa, 2003-2004) to 2520 per 100000 population/ year (children $<5$ years old, Ghana, 2007-2009) [5"'], with highest incidences in those infected with HIV, in patients with sickle cell disease, in young children, and in those residing in rural settings [5"']. The prevalence of NTS-related community-acquired bacteremia ranged from $8 \%$ in Nigeria and South Africa to $45 \%$ in the Central African Republic [ ${ }^{-"}$ ], with an overall case fatality rate of $20.6 \%$ (548 deaths/2656 iNTS disease cases) [5"'].

The emergence of iNTS organisms in Africa exhibiting resistance to various commonly used antimicrobials, including chloramphenicol, ampicillin, and co-trimoxazole, has been reported $[2 " ', 4 ", 15,16]$. These 'baseline' antimicrobial resistance profiles have been followed by the advent of resistance against third-generation cephalosporins; iNTS isolates with resistance to ceftriaxone has now been reported in the Democratic Republic of the Congo (DRC) $[17,18]$, Kenya $[19,20]$, Malawi $[15,21]$ and South Africa [2"'].

The Typhoid Fever Surveillance in Africa Program (TSAP), a population-based surveillance, conducted at 13 sentinel sites in 10 countries (Burkina Faso, Ethiopia, Ghana, Guinea-Bissau, Kenya, Madagascar, Senegal, South Africa, Sudan, and Tanzania) during 2010-2014, revealed an overall iNTS disease prevalence of $17 \%(94 / 568)$ among those with bacteremia [22]. The serovars S. Typhimurium (40\%, 38 out of 94 NTS positive cases), S. Enteritidis (12\%, 11 out of 94 NTS positive cases) and S. Dublin (11\%, 10/94 NTS positive cases) were the most prevalent [22-24], which is largely concordant with findings reported by Crump and Heyderman [4"] and Uche et al. [5"']. In TSAP, the adjusted incidences of iNTS disease were highest among children aged more than 1 year, ranging from 291 (95\%CI 176-482) per 100000 person-years-of-observations (PYO) (Guinea-Bissau) to 1733 (95\%CI 1373-2188) per 100000 PYO (Ghana) [22]. The iNTS disease incidences among children aged 2 to 4 years ranged from 49 (95\%CI 7-348) per 100000 PYO in Kenya to 1908 (95\%CI 1469-2479) per 100000 PYO in Ghana [22]. In addition, several independent reports on blood-culture-based surveillance data have shown that iNTS disease is present in other locations in Africa such as the DRC [18], the Gambia [25], and Ghana $[26,27]$. Data from different sites in Kenya found an incidence of 4134 per 100000 person-years [20] and 174 per 100000 person-years [28] in infants. Children under 5 years of age had an overall incidence of 36.6 per 100000 person-years [28,29]; incidences among children less than 5 years of age differed considerably by setting (rural setting: 3914 per 100000 person-years, urban setting: 997.9 per 100000 person-years) [30]. The presence of iNTS disease has also been reported from Mali [31], Mozambique where two studies were conducted [predominantly ST313 isolates [32]; infant incidence: 217.7 per 100000 child-years [33"]], and South Africa [34"'].

In contrast to Africa, the epidemiology of iNTS disease and corresponding antimicrobial susceptibility patterns are poorly described in Asia and South America, suggesting either a lower disease burden or a lack of epidemiological reporting. A multicenter, hospital-based study investigating communityacquired bacteremia in Indonesia, Thailand, and 
Vietnam from 2013 to 2015 identified an overall NTS-associated bacterial positivity rates of $27.5 \%$ (11/40 bacteremia cases) in children and $11.7 \%$ (7/60 bacteremia cases) in adults [35]. Limited iNTS prevalence (20/12940 bacteremia patients) and a $25 \%$ case fatality were reported among bacteremic patients hospitalized from 2009 to 2013 in Bangladesh [36]. A longitudinal study of communityacquired bacteremia in hospitalized children conducted in Malaysia from 2001 to 2011 found an iNTS prevalence of $16.2 \%(36 / 222)$, with most NTS isolated from infants below 1 year of age [37]. A surveillance study from Colombia investigated a sample of $4010 \mathrm{~S}$. Enterica isolates collected from blood and feces samples and found that $32.5 \%$ were S. Typhimurium, $28.2 \%$ were S. Enteritidis, and $2.9 \%$ were $S$. Dublin cases over a 6-year period [38]. These numbers are considerably lower than those reported from Asia and sub-Saharan Africa. Notably, S. Typhimurium ST313 variants have been isolated from humans and poultry in Brazil [39]. On the basis of the investigations of Almeida et al. [39], the organisms identified appear genetically distinct from the ST313 variants isolated in sub-Saharan Africa.

\section{HOST-ASSOCIATED FACTORS}

Common factors contributing to iNTS disease include extremes in age, the occurrence of immunosuppressive conditions, and other underlying comorbidities (e.g., diabetes, cancer, and cardiovascular diseases) [40]. In addition, climatic conditions such as increased rainfall or drought that can result in food scarcity, leading to malnutrition and increased transmission of malaria parasites are factors that may favor the transmission of NTS organisms [7"-]. Particularly in Africa, the association of iNTS disease with malnutrition (OR 1.44-2.42) and sickle cell disease (OR 35.6) has been described predominantly in children, whereas Plasmodium falciparum malaria (OR 1.5-4.1), anemia, and HIV infection (OR 3.2-48.2) are risk factors that are not generally associated with age $\left[2^{\mathbf{*}}, 4^{\mathbf{*}}, 5^{\mathbf{*}}, 7^{\mathbf{*}}, 24,29,41^{\mathbf{*}}\right]$. Adjusted odds ratios of 4.0 and 5.0 were calculated for the association of iNTS disease with moderate and severe anemia, respectively [33"]. Keddy et al. [34"-] found a significant association between an increased usage of antiretroviral therapy and a decrease in incident iNTS disease infections $(P<0.001)$ in a South African province. Similar observations were made by Lan et al. [42] in Vietnam. ST313, the most common $S$. Typhimurium variant associated with iNTS disease, was initially identified in HIVinfected patients [2"']. In comparison to other S. Typhimurium types (e.g., ST19), the genomically degraded ST313 may cause systemic infections and induce a lower inflammatory reaction in the intestine, exerted by evasion mechanisms from the immune response $[43,44]$. The genomic degradation includes the downregulation of gene expression involved in active cell invasion through effector proteins $[43,44]$. The reduced activation of macrophages is assumed to be caused by lower flagellin expression $[43,44]$. The survival time and replication rate were found to be more efficient in the investigated ST313 isolates compared with ST19 $[43,44]$. Therefore, the ST313 phenotype appears to become closer to that of typhoidal Salmonella, suggesting analogical adaptation toward a more invasive phenotype in humans [1"'].

In addition, an MDR phenotype may allow for rapid ST313 dissemination throughout susceptible populations [2"']. Advanced HIV disease leads to a reduced immune response in the gastrointestinal mucosa and poses a higher invasion risk of iNTS [7"']. Changes in the gastrointestinal microbiota, induced by the intake of acid blockers, gastric surgery, and antimicrobial pretreatment, are also suggested to favor iNTS disease $\left[7^{\mathbf{*}}, 45,46\right]$. Martz et al. [46] found stabilizing effects on the gastrointestinal microbiome associated with the ingestion of probiotics in mice, which may improve the functionality of the intestinal barrier.

\section{VACCINE DEVELOPMENT}

Effective vaccines preventing iNTS disease are likely to differ inherently from those protecting against S. Typhi infections. Studies from Africa have shown that naturally acquired antibodies against NTS correspond with a reduced risk of iNTS disease $[47,48]$. Several vaccine candidates targeting $S$. Typhimurium and S. Dublin are currently under development, some of which may provide protection against both serovars. The current status of iNTS vaccine considerations has been described in a recent review [49"']: several potential iNTS vaccines are under development, including live-attenuated, subunit-based, and recombinant antigen-based substances. Both humoral and cellular immunities are likely required to achieve full protection against iNTS disease. Liveattenuated vaccines provide both types of immune response; however, they may pose a risk for immunocompromised individuals [41"']. Inactivated iNTS vaccines may induce humoral immunity only and suppress NTS during the acute phase of infection, but are likely not to achieve systematic clearance in infected individuals [41"']. The lack of disease burden data from Asia and South America, coupled with the enormous number of NTS serovars, and the role of alternate prevention measures (i.e., access to improved water and sanitation and food safety) have contributed to a delay and a lack of investment in the development of iNTS disease vaccines. 
Previously, when animals were considered to be the only reservoir of NTS organisms, the implementation of hygiene and safety measures along a regulated and appropriate food chain was thought to be sufficient for the reduction of iNTS transmission. However, with the speculation that humans may be a growing alternative reservoir for ST313 [50], the development and deployment of iNTS disease vaccines appear to be a more viable solution. However, iNTS disease vaccines would not only require considerable funding to progress existing vaccine candidates, but also will require parallel vaccine deployment strategies to identify appropriate target age groups, schedules, formulations, and potential vaccine adjuvants.

An iNTS disease vaccine would need to be administered in infants to ensure protection before the peak occurrence of disease. This strategy, however, poses the challenge of combining iNTS and S. typhi vaccines, as the peak disease incidence for typhoid (5-8 years of age) is later than that for iNTS disease [51], except in highly endemic areas. A potential byproduct after the widespread use of a bivalent iNTS vaccine conferring protection against $S$. Typhimurium and $S$. Enteritidis would be serovar replacement by other Salmonella variants, such as S. Dublin. Such a serotype replacement was observed following the MenAfriVac campaign, when large populations in the African meningitis belt were vaccinated against Neisseria meningitidis serotype A and other serotypes subsequently emerged [52-54]. Another consideration would be a combined iNTS disease/malaria vaccine; this approach may be particularly prudent, given that malaria is associated with the severity of iNTS disease. Such a vaccine would then be tailormade for sub-Saharan Africa, but may be less applicable for low and nonendemic malaria regions (i.e., Brazil) [39]. This would potentially suggest the need to develop an independent, nonmalaria-combined vaccine that is applicable to all iNTS endemic regions.

\section{CONCLUSION}

iNTS is a major public health issue in sub-Saharan Africa. ST313 appears to be better adapted to humans than other S. Typhimurium, is associated with an increased disease severity, and has acquired an MDR phenotype. Observations of the Brazilian ST313 lead to some insights on this serotype that are also relevant for Africa. First, ST313 has the ability to arise in new locations independently and does not appear to be confined to sub-Saharan Africa. The fact that some Brazilian ST313 isolates exhibit different antimicrobial susceptibility profiles in comparison to African variants suggests that iNTS disease has the potential to evolve de novo outside of Africa, which may result in new and unlinked epidemics. Improvement in water and sanitation, a reduction in malaria incidences and malnutrition and improved management of HIV infections should additionally prevent iNTS disease from becoming an even bigger global health threat. However, the rapid emergence of ST313, the possible de-novo occurrence and spread of the future MDR and pathogenic variants place iNTS disease increasingly on the vaccine development agenda. Now is also a prime time to invest in enhanced iNTS disease surveillance. This enhanced surveillance is particularly important in Asia and Latin America, and is required to assess the actual extent of disease in these locations. Typhoid fever should be used as an example, when, despite the availability of vaccines, a lack of appropriate disease burden data stalled the global commitment, resulting in limited vaccine uptake and dampened efforts to develop conjugated vaccines. The persistence of typhoid fever culminated in the evolution of a highly antimicrobial resistant $S$. Typhi genotype (H58), which is spreading globally [55].

\section{Acknowledgements}

International Vaccine Institute (IVI) acknowledges its donors, including the Republic of Korea and the Swedish International Development Cooperation Agency. S.B. is a Sir Henry Dale Fellow, jointly funded by the Wellcome Trust and the Royal Society (100087/Z/12/Z). We are grateful to John Crump for his critical feedback on the manuscript.

\section{Financial support and sponsorship}

The authors A.H.H., U.P. and F.M. are supported through research grants by the Bill \& Melinda Gates Foundation (OPPGH5321 and OPP1127988).

\section{Conflicts of interest}

The sponsors had no role in study design, data collection and analysis, decision to publish, or preparation of the manuscript.

The findings and conclusions contained within are our own and do not necessarily reflect positions or policies of the IVI.

The authors declare no conflicts of interest.

\section{REFERENCES AND RECOMMENDED}

\section{READING}

Papers of particular interest, published within the annual period of review, have been highlighted as:

- of special interest

- of outstanding interest

1. Singletary LA, Karlinsey JE, Libby SJ, et al. Loss of multicellular behavior in - epidemic African nontyphoidal Salmonella enterica serovar typhimurium ST313 Strain D23580. MBio 2016; 7:02265-02315.

This article provides an update on the adapted selection advantages of the ST313 genome. 
2. Mahon BE, Fields PI. Invasive infections with nontyphoidal Salmonella in Sub-

- Saharan Africa. Microbiol Spectr 2016; 4:0015-2016.

Authors deepen the evidence on risk factors, including antimicrobial resistance patterns, and discuss vaccine candidates.

3. Wikswo ME, Kambhampati A, Shioda K, et al. Outbreaks of acute gastroenteritis transmitted by person-to-person contact, environmental contamination, and unknown modes of transmission-United States. MMWR Surveill Summ 2015; 64:1-16.

4. Crump JA, Heyderman RS. A perspective on invasive Salmonella disease in

- Africa. Clin Infect Dis 2015; $1:$ S235-S240.

Authors highlight the disease burden, antimicrobial resistance patterns, and transmission modes of NTS.

5. Uche IV, MacLennan CA, Saul A. A systematic review of the incidence, risk

- factors and case fatality rates of invasive nontyphoidal Salmonella (iNTS) disease in Africa (1966 to 2014). PLoS Negl Trop Dis 2017; 11:e0005118.

A comprehensive overview on the iNTS disease incidence as well as risk factors

and mortality across African countries.

6. Okoro CK, Barquist L, Connor TR, et al. Signatures of adaptation in human

- invasive Salmonella typhimurium ST313 populations from sub-Saharan Africa. PLoS Negl Trop Dis 2015; 9:e0003970.

The authors provide comprehensive phenotypic as well as genotypic data on ST313.

7. Crump JA, Sjolund-Karlsson M, Gordon MA, Parry CM. Epidemiology, clinical

- presentation, laboratory diagnosis, antimicrobial resistance, and antimicrobial management of invasive Salmonella infections. Clin Microbiol Rev 2015; 28:901-937.

Authors presented a broad and in-depth overview on the clinical picture as well as host-related risk and protective effects on iNTS disease.

8. Gilchrist JJ, MacLennan CA, Hill AV. Genetic susceptibility to invasive

Salmonella disease. Nat Rev Immunol 2015; 15:452-563.

The publication provides a comprehensive update on the global iNTS disease distribution

9. Ao TT, Feasey NA, Gordon MA, et al. Global burden of invasive nontyphoidal Salmonella disease, 2010(1). Emerg Infect Dis 2015; 21.

10. Graham SM, English M. Nontyphoidal Salmonellae: a management challenge for children with community-acquired invasive disease in tropical African countries. Lancet 2009; 373:267-269.

11. Hawkey PM, Livermore DM. Carbapenem antibiotics for serious infections. BMJ 2012; 344:e3236.

12. Kingsley RA, Msefula CL, Thomson NR, et al. Epidemic multiple drug resistant Salmonella typhimurium causing invasive disease in sub-Saharan Africa have a distinct genotype. Genome Res 2009; 19:2279-2287.

13. Keddy $\mathrm{KH}$, Sooka A, Musekiwa $A$, et al. Clinical and microbiological features of Salmonella meningitis in a South African Population, 2003-2013. Clin Infect Dis 2015; 1:S272-S282.

14. Lokken KL, Walker GT, Tsolis RM. Disseminated infections with antibiotic-

- resistant nontyphoidal Salmonella strains: contributions of host and pathogen factors. Pathog Dis 2016; 74:; ftw103.

This article describes the differences and similarities of the ST313 and ST19 phenotypes in detail.

15. Gordon MA, Graham SM, Walsh AL, et al. Epidemics of invasive Salmonella enterica serovar enteritidis and $S$. enterica serovar typhimurium infection associated with multidrug resistance among adults and children in Malawi. Clin Infect Dis 2008; 46:963-969.

16. Kariuki S, Revathi $\mathrm{G}$, Kariuki N, et al. Characterisation of community acquired nontyphoidal Salmonella from bacteraemia and diarrhoeal infections in children admitted to hospital in Nairobi, Kenya. BMC Microbiol 2006; 6:101.

17. Lunguya $\mathrm{O}$, Lejon $\mathrm{V}$, Phoba MF, et al. Antimicrobial resistance in invasive nontyphoid Salmonella from the Democratic Republic of the Congo: emergence of decreased fluoroquinolone susceptibility and extended-spectrum beta lactamases. PLoS Negl Trop Dis 2013; 7:14.

18. Kalonji LM, Post A, Phoba MF, et al. Invasive Salmonella infections at multiple surveillance sites in the Democratic Republic of the Congo, 2011-2014. Clin Infect Dis 2015; 1:S346-S353.

19. Kariuki S, Gordon MA, Feasey N, Parry CM. Antimicrobial resistance and management of invasive Salmonella disease. Vaccine 2015; 19:23.

20. Oneko M, Kariuki S, Muturi-Kioi V, et al. Emergence of community-acquired, multidrug-resistant invasive nontyphoidal Salmonella disease in rural Western Kenya, 2009-2013. Clin Infect Dis 2015; 1:S310-S316.

21. Msefula CL, Kingsley RA, Gordon MA, et al. Genotypic homogeneity of multidrug resistant $S$. Typhimurium infecting distinct adult and childhood susceptibility groups in Blantyre, Malawi. PLoS One 2012; 7:27.

22. Marks $F$, von Kalckreuth $V$, Aaby $P$, et al. Incidence of invasive Salmonella disease in sub-Saharan Africa: a multicentre population-based surveillance study. Lancet Glob Health 2017; 5:e310-e323.

23. Al-Emran HM, Krumkamp R, Dekker DM, et al. Validation and identification of invasive Salmonella serotypes in Sub-Saharan Africa by multiplex polymerase chain reaction. Clin Infect Dis 2016; 15:S80-S82.

24. Park SE, Pak GD, Aaby $P$, et al. The relationship between invasive nontyphoidal Salmonella disease, other bacterial bloodstream infections, and malaria in Sub-Saharan Africa. Clin Infect Dis 2016; 15:S23-S31.

25. Kwambana-Adams $B$, Darboe $S$, Nabwera $H$, et al. Salmonella infections in the Gambia, 2005-2015. Clin Infect Dis 2015; 1:S354-S362.
26. Eibach D, Al-Emran HM, Dekker DM, et al. The emergence of reduced ciprofloxacin susceptibility in Salmonella enterica causing bloodstream infections in rural Ghana. Clin Infect Dis 2016; 15:S32-S36.

27. Andoh LA, Ahmed S, Olsen JE, et al. Prevalence and characterization of Salmonella among humans in Ghana. Trop Med Health 2017; 45:3.

28. Muthumbi E, Morpeth SC, Ooko M, et al. Invasive salmonellosis in Kilifi, Kenya. Clin Infect Dis 2015; 1 :S290-S301.

29. Kariuki S, Onsare RS. Epidemiology and genomics of invasive nontyphoidal Salmonella infections in Kenya. Clin Infect Dis 2015; 1:S317-S324.

30. Verani JR, Toroitich S, Auko J, et al. Burden of invasive nontyphoidal Salmonella disease in a rural and urban site in Kenya, 2009-2014. Clin Infect Dis 2015; 61(Suppl 4):S302-S309.

31. Tapia MD, Tennant SM, Bornstein $\mathrm{K}$, et al. Invasive nontyphoidal Salmonella infections among children in Mali, 2002-2014: microbiological and epidemiologic features guide vaccine development. Clin Infect Dis 2015; 1:S332S338.

32. Moon TD, Johnson M, Foster MA, et al. Identification of invasive Salmonella enterica serovar typhimurium ST313 in ambulatory HIV-infected adults in Mozambique. J Glob Infect Dis 2015; 7:139-142.

33. Mandomando I, Bassat $Q$, Sigauque $B$, et al. Invasive Salmonella infections among children from rural Mozambique, 2001-2014. Clin Infect Dis 2015; 1:S339-S345.

This study provides evidence on causal relationships between iNTS diseased and their host factors.

34. Keddy $\mathrm{KH}$, Takuva S, Musekiwa A, et al. An association between decreasing

- incidence of invasive nontyphoidal salmonellosis and increased use of antiretroviral therapy, Gauteng Province, South Africa, 2003-2013. PLoS One 2017; 12:e0173091.

Most recent findings which suggest that antiretroviral therapy may be an important factor on the occurrence of incident NTS infections.

35. SAIDCR Network. Causes and outcomes of sepsis in southeast Asia: a multinational multicentre cross-sectional study. Lancet Glob Health 2017; 5:e157-e167.

36. Shahunja KM, Leung DT, Ahmed T, et al. Factors associated with nontyphoidal Salmonella bacteremia versus typhoidal Salmonella bacteremia in patients presenting for care in an urban diarrheal disease hospital in Bangladesh. PLoS Negl Trop Dis 2015; 9:e0004066.

37. Nor Azizah A, Fadzilah MN, Mariam M, et al. Community-acquired bacteremia in paediatrics: epidemiology, aetiology and patterns of antimicrobial resistance in a tertiary care centre, Malaysia. Med J Malaysia 2016; 71:117-121.

38. Rodriguez EC, Diaz-Guevara $P$, Moreno J, et al. Laboratory surveillance of Salmonella enterica from human clinical cases in Colombia 2005-2011. Enferm Infecc Microbiol Clin 2016; 30:30008-30018.

39. Almeida F, Seribelli $A A$, da Silva $P$, et al. Multilocus sequence typing of Salmonella typhimurium reveals the presence of the highly invasive ST313 in Brazil. Infect Genet Evol 2017; 51:41-44.

40. Turgeon P, Murray R, Nesbitt A. Hospitalizations associated with salmonellosis among seniors in Canada, 2000-2010. Epidemiol Infect 2017; 145: 1527-1534.

41. Mastroeni $P$, Rossi O. Immunology, epidemiology and mathematical modelling

- towards a better understanding of invasive nontyphoidal Salmonella disease and rational vaccination approaches. Expert Rev Vaccines 2016; 15:15451555.

The authors address necessary considerations related to the vaccine development and introduction in different populations.

42. Phu Huong Lan N, Le Thi Phuong T, Nguyen Huu H, et al. Invasive nontyphoidal Salmonella infections in Asia: clinical observations, disease outcome and dominant serovars from an infectious disease hospital in Vietnam. PLoS Negl Trop Dis 2016; 10:e0004857.

43. Carden S, Okoro C, Dougan G, Monack D. Nontyphoidal Salmonella typhimurium ST313 isolates that cause bacteremia in humans stimulate less inflammasome activation than ST19 isolates associated with gastroenteritis. Pathog Dis 2015; 73:24.

44. Ramachandran G, Perkins DJ, Schmidlein PJ, et al. Invasive Salmonella typhimurium ST313 with naturally attenuated flagellin elicits reduced inflammation and replicates within macrophages. PLoS Negl Trop Dis 2015; 9:e3394.

45. Freeman $\mathrm{R}$, Dabrera $\mathrm{G}$, Lane $\mathrm{C}$, et al. Association between use of proton pump inhibitors and nontyphoidal salmonellosis identified following investigation into an outbreak of Salmonella mikawasima in the UK, 2013. Epidemiol Infect 2016; 144:968-975.

46. Martz SL, McDonald JA, Sun J, et al. Administration of defined microbiota is protective in a murine Salmonella infection model. Sci Rep 2015; 5:16094.

47. Gordon MA. Invasive nontyphoidal Salmonella disease: epidemiology, pathogenesis and diagnosis. Curr Opin Infect Dis 2011; 24:484-489.

48. MacLennan CA, Gondwe EN, Msefula CL, et al. The neglected role of antibody in protection against bacteremia caused by nontyphoidal strains of Salmonella in African children. J Clin Invest 2008; 118:1553-1562.

49. Tennant SM, MacLennan CA, Simon R, et al. Nontyphoidal Salmonella

- disease: current status of vaccine research and development. Vaccine 2016; 34:2907-2910.

This article discusses potential vaccine candidates and immune responses in different vaccine-receiving populations. 
50. Kariuki S, Revathi G, Kariuki N, et al. Invasive multidrug-resistant nontyphoidal Salmonella infections in Africa: zoonotic or anthroponotic transmission? J Med Microbiol 2006; 55(Pt 5):585-591.

51. von Kalckreuth $V$, Konings $F$, Aaby $P$, et al. The Typhoid Fever Surveillance in Africa Program (TSAP): clinical, diagnostic, and epidemiological methodologies. Clin Infect Dis 2016; 62(Suppl 1):S9-S16.

52. Mohammed I, lliyasu G, Habib A G. Emergence and control of epidemic meningococcal meningitis in sub-Saharan Africa. Pathog Glob Health 2017; $111: 1-6$
53. Collard JM, Issaka $B$, Zaneidou $M$, et al. Epidemiological changes in meningococcal meningitis in Niger from 2008 to 2011 and the impact of vaccination. BMC Infect Dis 2013; 13:1471-2334.

54. MacNeil JR, Medah I, Koussoube D, et al. Neisseria meningitidis serogroup W, Burkina Faso, 2012. Emerg Infect Dis 2014; 20:394399.

55. Wong VK, Baker S, Connor TR, et al. An extended genotyping framework for Salmonella enterica serovar typhi, the cause of human typhoid. Nat Commun 2016; 7:12827. 